\title{
IMAGE-MAKING AND ARCHITECTURE: A DIGITAL MEDIUM FOR QUALITATIVE DESIGN REPRESENTATION
}

\author{
Dewa Ayu Ketut Septika ${ }^{1 *}$ \\ ${ }^{1}$ Faculty of Art and Design, Bandung Institute of Technology, Indonesia
}

\begin{abstract}
Architecture is inseparable from visual aspects in the form of representation as a way to establish communication. Due to digitalization and the rapid development of technology, there has been a shift in the paradigm of image representation. Manual images become digital images, from sketching and drawing to image-making and rendering. Digital rendering is considered to be object-oriented and quantitative. It has the characteristics of being precise, fast, reflecting form and materiality, with a photorealistic image as a result. There is no visible involvement of subjects such as the architect, image-maker, and viewers in the process because the image represents the final outcome. Here, the role of representation to evoke imagination and deeper interpretation is lost because it does not leave room for intervention and contemplation of the design. That is why another kind of digital method emerged in the world of representation; instead of "rendering" the design as an image, it is relevant to the act of "making" an image as design representation. Its characteristic as a representation method makes the output images have the ability to be evocative in order to make its viewers contemplate and interpret the design, which makes it a qualitative representation. The aim of this paper is to understand the act of image-making through digital collage as a medium for qualitative representation in architectural design. By comparing digital rendering and collage images obtained through literature studies, this paper wants to offer the author's viewpoint on the qualities and experiences brought by architectural representation.
\end{abstract}

ARTICLE INFO

Keywords:

Architecture, Collage, Image-making, Qualitative, Representation

\section{Introduction (Ebrima Bold 10)}

Designers in all disciplines live in a very visual world, architect and other practice in the design fields generally work with the visual medium. Unlike artists, who can directly work with their idea of mind in the form of objects, architects are persistently faced with the difficulty of not being able to work directly on the architectural object of creation, but always having to work through a visual medium of representation (Bernath, 2007: 37). This medium shares an internal function to serve as a means of exploration and notation within the body of the architect's practice. Externally, they become a medium for communication, to others in the practice and the public (Riahi, 2017: 815). This visual medium comes in the form of drawings, such as plan and section, that focused on measurements, makes them quantitative representation. Meanwhile, along with them came perspective drawing which is known to represent the architect's idea and intention of the design through a depiction of building's experiences. These qualities of perspective drawings categorized them to qualitative representation. Therefore, quantitative representation shows the measurable (visible) information and qualitative representation shows experiential (invisible) experiences of the design.

Many methods of design representation are here thanks to what the digital computational abilities brought to us, shifting the act of manual representation, i.e., drawing, to digital rendering and image-making. This digital act of design representation has become a part of today's architectural process. However, now digital rendering has been deemed as a merely commercial commodity to sell the design and considered too banal to reflect the true essence of architectural idea (Bernath, 2007: 49). Thus, perspective drawing produced through digital rendering cannot serve its role as a qualitative representation.

That is the reason why architects have been searching for alternative ways to represent their design ideas digitally. The act of rendering an image slowly becomes the act of image-making. This paper wants to address whether digital representations, specifically perspectives produced 
through the act of image-making, are still embodying the qualities like its predecessor, the conventional handdrawing perspectives. Furthermore, whether it serves its function as a qualitative representation medium that evokes deeper interpretation and exploration of design ideas.

\section{Literature Review}

\subsection{Qualities of Representation}

As mentioned earlier, designers live in a visual world and often sensitive to the appearance of artefacts and environments around them. Thus, the visual qualities of their design products are, with practically no exception, of great importance to them, as well as to clients and users (Goldschmidt on Idi, 2007: 5). Idi (2007: 5) explained that the process of growing or developing the idea is vital to idea perfection. The way to achieve that perfection (idealistic visual) is through an exploration of design ideas continuously, in order to find what is considered the best solution and its alternatives.

Initial ideas and design processes are not only existed in the mind of the designer but are present and expressed in the real world, making them a form of an artefact as well. Specific in the realm of architecture, an architect expressed their idea of mind in the form of text or visual representation. Architects think of the solution to the problem and generate it through a design idea, then present it to their design team, a client, and, if so, to the public audiences. Normally, architects use drawing to execute their ideas because it is indeed fast. The other parties can identify and relate to the design; in other words, they can communicate easily through a drawing. Drawings compose a powerful articulation of the formation and development of thoughts and intentions in architecture (Riahi, 2017: 816). It can describe the design as a conversation, dialogue between the designers and themselves, or the people involved in the design process. Therefore, this process and the outcome of representation can reflect not only a single thought, but also the intervention of subject involved.

Relevant to its characteristics, drawing becomes a tool for developing even generating new ideas in the design process. Due to its quick, unfinished nature and its flexibility to accept intervention and evaluation, it makes a drawing left open to more interpretation of thought, allowing design alternatives to be explored. Designer according to Goel (on Suarez, 2015: 48), exploring ideas through the act of drawing, "...where movement is from one idea to a slightly different idea rather than a more detailed version of the same idea."

Drawings as an architectural representation play visual aspects to depict the experience of space or building. What is depicted on paper, then, are not "realities" but experiences of objects (Suarez, 2015: 48). While a work of architecture is presented to us directly, a representation shows it indirectly through various experiences in its imagery. Very often, imagery experiences are understood by their subjects as echoes, copies, or reconstructions of actual perceptual experiences from their past; at other times, they may seem to anticipate possible, often desired or feared, future experiences (Idi, 2010: 6). Drawings must be able to connect the experience produced by the drawings with the experience that are actually produced by the physical object that is taken as the reference. Architectural representation in the design process depicts a referent that is unbuilt, yet. That is why the experiences that are represented are intentional experiences, planned by the architect.

Then we can say that drawings as representation is referential and always fictional, also has a role as a tool for exploration and communication, embodying the thoughts and intentions of subjects involved.

There are two aspects that constructed a representation: the object represented (the what) and the representational medium (the how). However, this paper will not discuss "the how" aspect because it is considered a technical element related to the techniques of image creation and not related to the purpose of this paper. Then, "the what" aspect will be the key to analyze images in the discussion later. The object represented is relevant to "what" the viewer received and experienced through the represented object in the drawing. Suarez describes (2015: 50) that "the what" aspect is referred to as the content, meaning, message, or subject depicted through visual elements.

$\begin{aligned} & \text { Table 1. The what aspect of a representation. } \\
& \text { Representation of } \\
& \text { experience (production) }\end{aligned}$
\begin{tabular}{ll} 
A representation includes & \multicolumn{1}{c}{ A representation (reception) } \\
only some aspects or elements & focuses the viewer's attention \\
of the referent and is thus: & $\begin{array}{l}\text { towards certain aspects of the } \\
\text { object and is thus: } \\
\text { selective }\end{array}$
\end{tabular}

Representation originates from past experiences; in other words, the references that the maker and viewer have. Because of that, representation has these qualities regarding the contents and references it contains:

- Selective quality, it is impossible to experience an object as it truly is. Experience itself is selective, and it is also limited by our view of the world. It establishes a point of view and frames the view that is presented.

- Directive quality, what is considered important, is represented, and what is represented gains importance. A representation has the ability to focus or direct the viewer's attention towards certain elements that are shown as being more important. They limit what is being portrayed and therefore dramatize certain aspects while muting or even altogether silencing others.

In representing architecture, the various elements that shape our experience of space or building are frozen in the form of an image. The elements are depicted in the drawing in the form of visual language (tangible), even visual-perception of depth and lighting, movements and activities in space, meanings, thoughts, and emotion (intangible) that architecture can make its user feel also depicted. Therefore, a drawing is not solely a matter of recording the visual essence of the object, but also the visual percept that delivers the entire sensual (including the visual) essence of it. 
The result from conventional representation, i.e., drawing, is often limited to how it can convey the essence of space or building experience. But recently, the development of digital technology has broadened the horizons of architectural representation. It has caused a shift in conventional representation towards digital representation; sketches become digital images; the act of drawing becomes rendering. Through digital visualization, conducted from the initial stage to the final stage of the design process, digital tools offer a new way of design representation, promising a faster and more realistic results.

\subsection{Digital Representation What is rendering?}

The digital technique, commonly known as rendering, creates photorealistic images that are currently in the center of attention in architectural practice. It offers an effective way of presenting an architectural project as if it were already part of reality. Instead of communicating design ideas by presenting plan and section drawing, rendering focused on showing space or building experiences through perspective drawing. Bernath stated in his paper regarding the act of rendering and its intrusive characteristics (2007: 50) that the perspective drawing has a higher intellectual significance than simply to capture a realistic view. Perspectives are more qualitative than quantitative because the experience of an environment or an object can be perceived directly from a perspective. These qualitative representations of architecture can be achieved by showing the appearance and experience of buildings (Suarez, 2015: 47).

Perspective drawings processed through rendering technique are constructed by atmospheric effects and visual stereotypes related to experiences which the viewers had in their minds from their past experiences, as stated before. That is why rendering can deliver a sense of the design that can be immediately understood and appreciated by the general public. Through this ability, rendering states its provocative power to stir affective responses in the mind of the perceiver (Bernath, 2007: 39).

Because of that reason, rendering is on high demand in architectural practice, and there is indeed a need to communicate the design idea through a realistic depiction that is relevant to the general public. But in renderings, design ideas are not only being represented but also manipulated. The consequence is that digital simulations become more dashing and less faithful to the object or idea represented.

In his paper, Bernath (2007: 38) stated that the overriding power of the rendering, awash with market expectations and popular imageries, competes with the specialized autonomy of the design process. In design practice, rendering should be done near or at the conclusive stage of the design development, but in fact, rendering is conducted at each stage of the design process. Because of that, architects often find themselves in situations where the rendering of the design is conducted ahead of design development. This action intrudes on the normal routine of the design process. The effort of design is then directed to support and fit into the projected vision from the rendering. In other words, the rendering is dominating over the design imagination, and it often surpasses the architectural content planned by the architect. How the exploitation of the rendering process is transforming the relationship between the commonly assumed hierarchy of the design idea and its representation.

Instead of being a by-product of the idea in the design process, rendering became the it-product of architecture for selling purposes. Bernath (2007: 49) lastly stated that therefore rendering exists as an afterthought of design; it is deemed as an advertisement; a commercial commodity aiming to seduce the viewers and sell the design. Due to that reason, rendering is considered too banal to reflect the true essence of the architectural idea, the experience embodied in the predicted space, and failed to serve its name as a qualitative representation.

As the digital practice of architecture exploits the possibilities of the same tools and techniques used in visualization, architects started to search for alternative representation methods that can describe the experiences and qualities of architectural propositions. Adopting ideas and intention from older compositing method, i.e., collage, architects are now pursuing some kind of imageries that actually accentuates not only about how it was produced and consumed but also about the qualities of the images as an architectural representation.

\section{What is image-making?}

Image-making is a form of resistance from the act of rendering. It proposes an alternative value different from digital rendering, which is efficiency and accuracy achieved through realistic images. Regarding this different act of producing architectural representation, based on what Ratinam stated (2012: 14, 85) that the distinctions of a quantitative and qualitative architecture representation lay on the information conveyed through the images. One is representing geometry through the measurable (visible) reading, and the other is representing narratives through experiential (invisible) experience depicted in the images.

From the way digital representation progressed, photorealistic renderings and literal flythrough become a conduit for only a narrow, almost empirical or quantitative understanding of architecture, failing to demonstrate its cultural significance and the ways in which architecture enriches our lives and daily experiences (Ratinam, 2012: 140). Thus, the main purpose of this act of image-making is to restore the role of perspective drawing that conveyed qualitative understanding including narratives and experiential experience, in the digital world.

Besides that, the process of image-making explores flexibility in editing so that they can serve as a tool of exploration and evaluation in the design process. Current 
image-making techniques are largely based in collagestyle work, imagery that is quickly generated by the use of "sourced" images. In contrast to render, the collages empower speculation rather than one-to-one. Therefore, the image becomes as much of the client as the architect where both can project and construct their own narratives (Horn, 2017: 29). Collage of images that are made digitally is the adapted technique in making representation images.

Collage has a role in constructing architectural ideas of form and space through visual narratives and stories. The story would go through a transitional process from a 2dimensional media into the spatial quality or a 3dimensional media, and then it is translated into architectural form (Rahayu, 2015: 1). This process causes ambiguity in the depiction of architectural ideas. The ambiguity in collage is the reason why there are speculation and a variety of perceptions in understanding the images, thus empowering the process of exploration of an architectural idea. Ambiguity would also allow a deeper understanding on existing potential in architecture and also the integrity of perception in communicating an idea within its translation (Rahayu, 2015: 1).

As an architectural tool, this wide-ranging medium mixes high and popular references and offers a dynamic, inventive connection to cultural context (Horn, 2017: 17). The ethos of collage shapes every aspect of contemporary culture, from the glut of signs and images to the many layers of digital information to the art of sampling (Horn, 17), making its visual so different from the rendering stereotype. Because of that, collage is considered only to construct a depiction of the aesthetic idealism of the creator and thus, is insufficient in representing architecture.

But now, the phenomena of digital collage in the architecture field has adapted to the normal routine of designing and empower the disciplinary shift of a digital work that is both illustrative and informational. Digital collage is one of the techniques used in the act of imagemaking nowadays, and collage images will be the object of discussion in this paper. "The what" aspect of architecture representation will be analyzed through a series of collage images to reveal whether this particular digital method can embody qualities of a qualitative representation.

\section{Research Method}

In this paper, images that serve the function as architecture representation will be analyzed. Based on the literature studies, an image can be called a qualitative representation if the image: (1) contained experiences and qualities (directive $\&$ selective) of the space based on the ideas and intention of the architect, (2) is a representation and depiction of "the what" aspect (the content, meaning, message, or subject depicted through visual elements), and (3) function as a medium for design exploration (as a process, not a by-product of a design).

The three points above serve as a parameter used in analyzing the image and its content. Referring to this, an interpretation of visual elements depicted in the image is conducted to find its content. Several images chosen are images produced through the two different methods of representation, collage images (result from the act of image-making) and photorealistic perspectives (result from the act of rendering).

First, an analysis of images produced through the act of image-making, namely digital collage technique, will be conducted in order to reveal the information and qualities contained in the images. This finding will become the basis for answering whether the collage image is the qualitative representation in the digital era. The images included are based on chronological considerations regarding the emergence of this technique as the act of image-making in architectural practice.

Superstudio is one of the early participants in collage culture in the architectural field. Office KGDVS, Fala Atelier, and duastudio are some of the many consultants who consistently create collages in their routine of architectural practice. The images in the discussion are one of their works that are considered relevant and is able to prove an important point based on the theory of qualitative representation.

Another analysis will be performed on the image produced through the act of rendering and is a work from the specialized architecture visualization studio. The rendered perspective from KUNKUN Visua/is considered fit into the description of the rendered images described in the previous section. The analysis will prove whether the perspective produced through a rendering process has somewhat become a quantitative representation. Then, the author will conclude the comparison between the quality of the images produced through two different methods.

Both analyses are conducted in order to understand if digital representations, specifically perspectives produced through the act of image-making, are still embodying the qualities like its predecessor, the conventional handdrawing perspectives. Furthermore, whether it serves its function as a medium for the exploration of the design. The discussion and writing of this paper are based on the literatures, quoted and listed.

\section{Results and Discussions}

\subsection{Ideas, Represented - Images, Representing}

In representing architecture, everything ends up as an image. But when you don't take time to contemplate the image and decipher the message, there is a shortage of attention after overdosing on visual material. An image is not that different from a text in this sense; you have to take some time and read it. It has a plot, a narrative, a logic of its own. It can go straight to the point, have some twists and jokes, some hidden meanings between the lines, be extremely serious or ironic, and playful. Like those qualities in a text, images in a collage manner contain selective and directive quality in order to represent elements that are important and avoid visual addiction.

The experience presented by representation is selective because of its inability to include all aspects of the 
experience of objects or spaces. The term selective referred to the decision of showing what elements the creator wants to show or accentuate (directive quality). There are some elements of spaces the architect wants to bring out on certain projects because not every project is different in their own way. Therefore, the images can be made to represent the architect's intent specifically on that project, like on Figure 1.

The interior space is filled with no information on the furniture layout, glossy materials, or anything that is usually portrayed in the photorealistic rendered images. The architect only wants to focus on showing the shadow play, the opening composition on the wall, and the light color and texture of the materials. Besides that, the image shows how those aspects of the interior could narrate and accentuate the space.

Not only that, like what is stated by Riahi (2017: 81), that drawings compose a powerful articulation of the formation and development of thoughts and intentions in architecture. It can describe a design as a conversation, or dialogue between the designers with the people involved in the design process, in this case of Figure 1, is the client.

Their client wants some kind of freedom on the design process and the design output; thus, the architect lets the client design the movable furniture layout by themselves, how they narrate their daily activities in that space based on their life phases as a human. And so, Figure 1 as representation is not only selective but also have the ability to evoke the client to make interpretation not only about the space but also their own interpretation of the future experience from the space.

On the contrary, Figure 2 shows how a daily view of a house looks like. It represents how the miscellaneous things and composed chaos in interior space are the ones that make a house felt more lived-in compared to the classic glossy rendering that scientifically describes bare and pristine spaces. It shows some uncertainty of the inside spaces and encourages the possible exploration of them.

Like what is stated by Goel (on Suarez, 2015: 48), that designer explores ideas through the act of drawing, "...where movement is from one idea to a slightly different idea rather than a more detailed version of the same idea." Even through the act of image-making i.e., collage, not drawing, the architect creates a series of images that may always question the possible experiences by introducing some wild juxtapositions to the everyday domestic space. Therefore, collage of fragments or images in Figure 2 can represent the architect's intention of the experience of the space and also allowing the viewer or user to make interpretation and exploration of the space represented.

To achieve those purposes (as a tool of exploration), architects use their power to direct the viewer's attention through the images. In figure 3 , isolating some of the visual stereotypes, the trees, by using a darker shade of color, it directs the viewer's attention to the other visual objects present in the image. In this case, the architect or image-

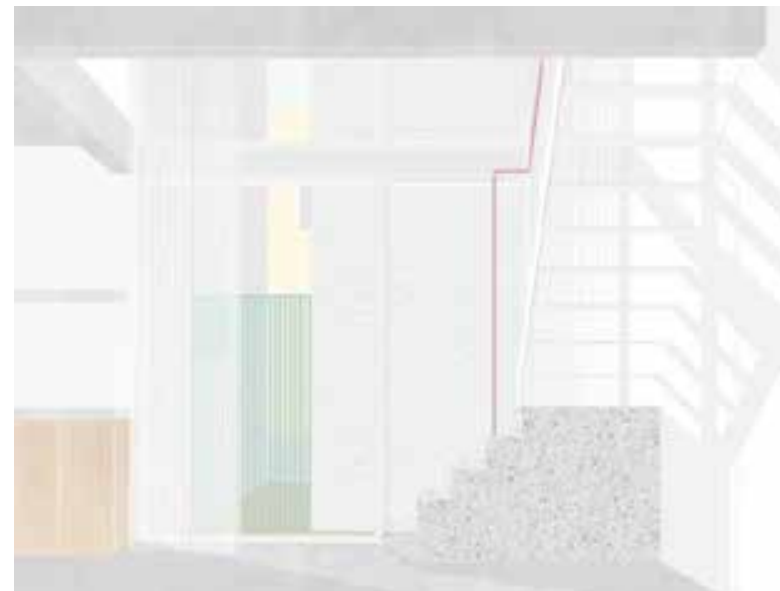

Figure 1. The alley, the void, the house by duastudio, 2020 Source: http://duaoffice.com

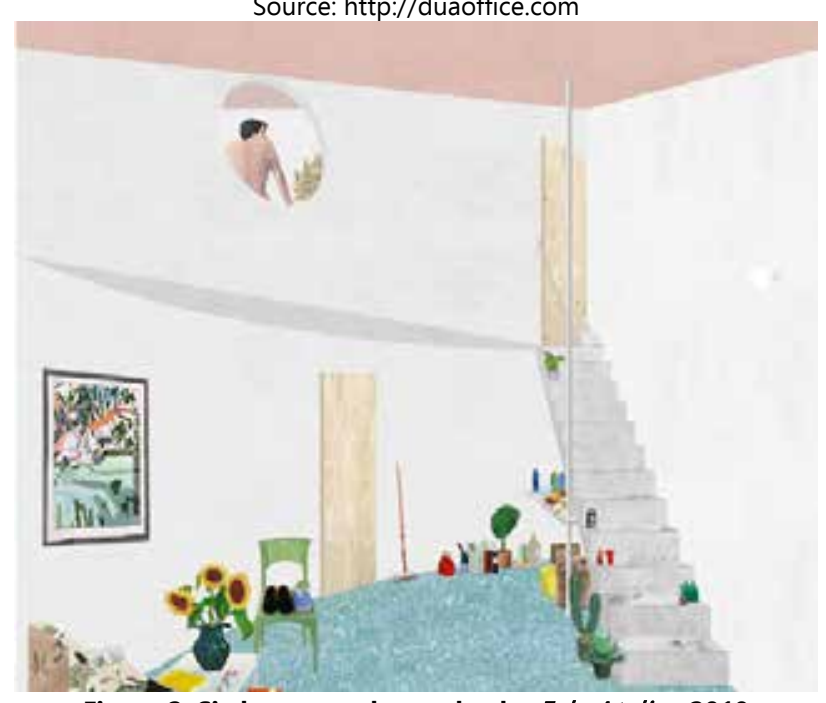

Figure 2. Six houses and a garden by Fala Atelier, 2019

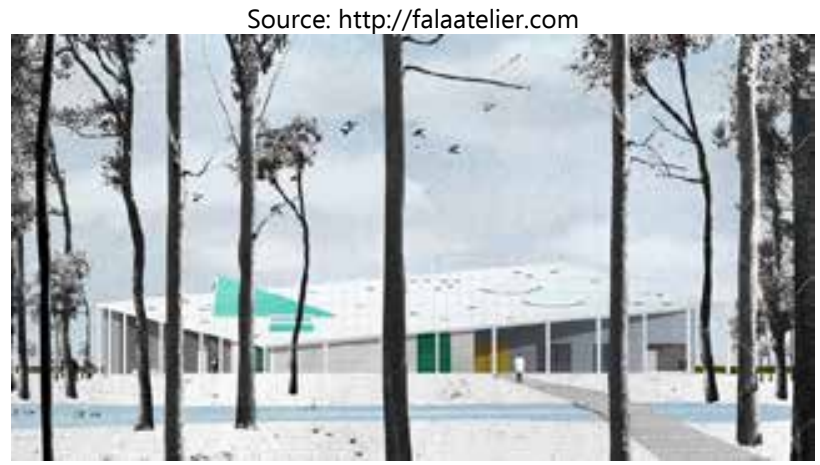

Figure 3. Crematorium by Office KGDVS, 2013

Source: http://officekgdvs.com

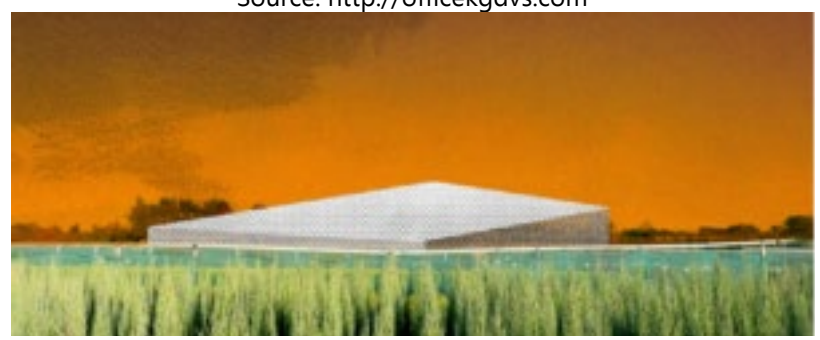

Figure 4. Drying Hall by Office KGDVS, 2013

Source: http://officekgdvs.com

maker intends to highlight the perceptual dissolution of a manmade environment where the building is surrounded by a forest. The difference in ground texture directs our attention to the footpath that led from the forest, across a river, and finally into the building, showing the accessibility 
of the space. Figure 3, as a representation, has an ability to direct the viewer's attention to the intentional aspects and experiences deemed to be more important, planned by the architect. The image not only shows the viewer what to look at but also tell the viewer how to look at it, thus make this image has the directive quality of a representation.

In contrast, by fading the building and not the environment, this representation in figure 4 told us that the building is there to be a statement and landmark in the middle of its site. The building is represented as something taller than the trees and can be seen from far away. This building is depicted as the most extreme and simple incarnation of a big box; it removes anything and even vaguely describes its function, a true building without content. The actual material of the building is perforated steel, and from the characteristic of the material, the perforations, represented by the opacity of the building. It gives the viewer the impression of an almost non-existing building, a building through which one can still see the silhouettes of the things inside, a building that does not land in the ground but floats in space. Figure 4 tells a story about a building that is questionable in function which allows the viewer to assume and imagine about the experience of the building in reality.

There is one thing in common depicted in this series of images that are made with collage technique: that ambiguity and meaning are not explicitly conveyed by the architect, thus allowing a free interpretation from the viewer. As stated by Rahayu (2015: 1), ambiguity in collage is the reason why there are speculation and variety of perceptions in understanding the images, thus empower the process of exploration and evaluation of an architectural idea. With that impact, collages would allow a deeper understanding of the existing potential in the represented architecture space.

Due to its intuitive and unfinished state, it allows the viewer to have time for thinking and interpret the drawing. An interpretive impact is important because it allows us to think beyond geometry, the intangible content of the images, and the experiences it contains. This act then generates deeper thinking and understanding of spaces that can help the viewer make a critical and contextual judgment of the design object or even the idea itself. Not only that, it can also help the architect to question every decision they made for the design and explore alternative ideas in easier sense and technique, keeping in mind that digital rendering demands sophisticated hardware and high software skill.

\subsection{Qualitative Design Representation}

These efforts to mirror a building before it is built, I would argue, only push architects further away (rather than closer to) understanding and representing the ephemeral and invisible qualities found in the eventual buildings and spaces that make them truly fulfilling (Ratinam, 2012: 7). As stated before, perspective representation made through rendering doesn't do justice to the architect's idea and the building experience conveyed by it.

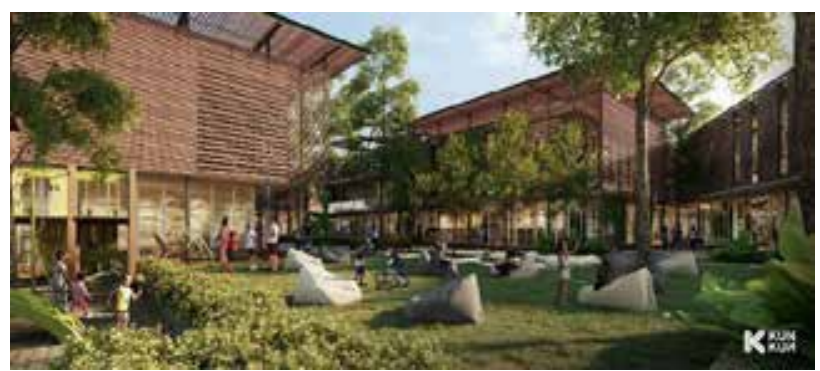

Figure 5. SWARNA project by KUNKUN Visual Source: http://kunkun3d.com

Rendering, like in figure 5, depicts a highly detailed, materially articulated, and populated architectural space. It mimics photographs and demands the architect and the viewer an understanding of the literacy of photographic material. It is not a hard thing to do because photography is trusted by people to capture a reality, as seen by the human's eyes. The criteria of this kind of representation are "reality" as defined by photographic language.

Suarez (2015: 48) warned about this issue, i.e., What is depicted on paper is not "realities" but experiences of objects. It could inherently enslave the architect and the viewer to trust that reality as the "true" reality, which is not because representation is always fictional.

Therefore, rendering images could dictate over the design beyond the control of the designer and dictate viewer expectations of physical reality. This is relevant to what Bernath (2007: 47) stated that rendering became the afterthought of design. Rendering became a representation that does not evoke the exploration of the design but instead dictates it.

Rendering usually needs sophisticated software and skills, and so it typically very expensive. Because of that reason, as a project document, they are difficult to update, making the act of rendering kind of discouraging. Some architects think that the process of representing in imaging should not be merely computational and relies on the software but instead should rely upon the other forms of knowledge, experience, imagination, and composition skill. Those qualities of perspective rendering then don't quite fit with the description of a qualitative representation.

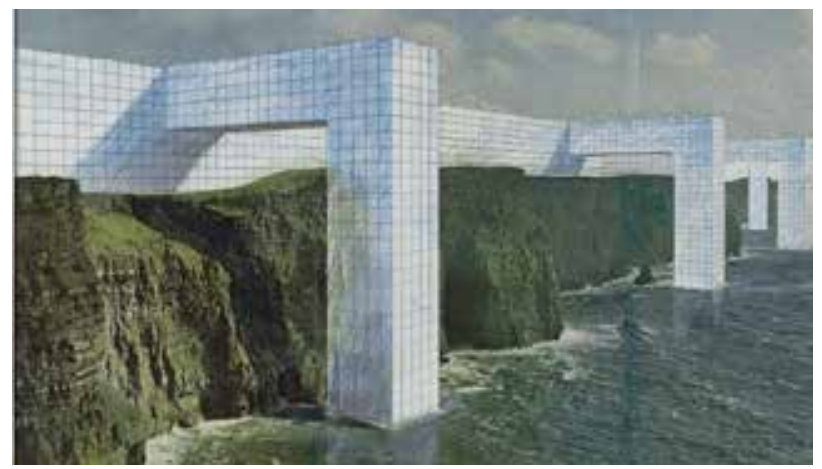

Figure 6. The Continuous Monument Project by Superstudio Source: http://socks-studio.com 
Superstudio is one of the participants in collage culture in the twentieth century that reflects on their architectural practice in an aesthetic and cultural way, often analytical and consists of a statement of a phenomenon. They created collage images that conjure architecture in a new way. One of them is figure 6 that represented their way of thinking; the urbanization that affected the global nature of the urban structure itself. The setting of the matter is on a critical level, presented as a model for interpretation and representing the generation through a series of analytical images. through collage is indeed served their role as a qualitative representation in the architecture realm.

\section{Conclusion}

A qualitative representation in architectural practice is a product of a design process that is capable of turning design ideas in the form of experience by depicting only some of its many possible qualities. Architectural representation should not be considered as something that is conducted at the final stage of the design process. Representation should have the ability to evoke the eyes

Table 2. The classification of architecture representation.

\begin{tabular}{|c|c|c|c|}
\hline Category & Experience/Intention & Representing/Depiction & Action \\
\hline Quantitative representation & $\begin{array}{l}\text { Preciseness } \\
\text { (Measured, detailed visual } \\
\text { element, one to one) }\end{array}$ & $\begin{array}{c}\text { Geometry } \\
\text { (Dimensions, technical detail, } \\
\text { materiality) }\end{array}$ & $\begin{array}{l}\text { Technical (document) drawing, } \\
\text { Rendering }\end{array}$ \\
\hline Qualitative representation & $\begin{array}{l}\text { Experiential } \\
\text { (Intangible, the impact of the } \\
\text { visual element, speculation) }\end{array}$ & $\begin{array}{c}\text { Narrative } \\
\text { (Content, meaning, message, } \\
\text { subject, story, intention, } \\
\text { statement) }\end{array}$ & $\begin{array}{l}\text { Drawing (conventional), } \\
\text { Image-making (collage, post- } \\
\text { production, animation) }\end{array}$ \\
\hline
\end{tabular}

In the collage, they focused on the design of monumental figures on a landscape scale. A form of design free of all functional meaning, the aim was to accentuate the aspect of fantasy and the lack of depiction technique. They emphasized the importance of the project focused on the underlying ideas of architecture rather than the object specifically. The image was a conceptual project, never meant to be built. The imagined material for the grid was glass, a symbol of industrial technology, equality, and clarity. The image shows the glass grid dominating Earth's topography through sheer neutrality and ubiquity. The grid counters narrative, which takes place in and through architecture in this imagined, context-less setting, there is no benchmark against which to measure normality or strangeness.

At that time, modernism hit the world and so superstudio wanted to imply the architecture of the time as the disruptive infrastructure of nature through collage images. The image is no longer a scale reduction of reality, charged with a functional purpose, but is presented as a conceptual fact. Along with that are the qualities of representation that is present in the image. The characteristics of the building are far different from the landscape in reality; the image represented the building as what it is, a fictional object that does not need detailing as much like the rendered drawing. The image is not trying to deceive its viewer into thinking it as the actual reality; it just needs to deliver the message and statement behind them. Those characteristics of the image are the selective and directive qualities describe in architectural representation. As for the space experience, figure 6 is trying to predict and, at the same time, insinuated the architectural condition of that time. With those series of images, we can say that image-making

and minds in order to be a tool of exploration,
experimentation, and reflection in the architectural process, which can be obtained through a qualitative method of depiction.

The qualitative term in representation is intended to embody the values, the experiences, and the qualities of an imaginative or built architectural design. There is a distinction between reading a drawing and experiencing a building. Experiences of the building are invoked through the images; they provide means to understanding qualities not available in the built work, not just variations of it.

The discussion in the previous section has confirmed the references and opinion stated that the rendering process impedes the exploration of design. Instead of becoming a reference in realizing an unbuilt space in reality, rendering dictates the unbuilt space to become the false reality that is deemed as perfect. The perspective rendering has shifted into advertisement purposes, to attract and seduce the audiences in the blink of an eye. But not through the presence of rich space experiences, and obviously not through contemplation and deeper interpretation of an architectural idea.

Nonetheless, as many kinds of representation are needed to convey various types of experiences of a space or a building, each representation has a particular way of experiencing the built environment. A representation that shows us all things with a similar level of detail, as happens with realistic rendering, is on the same page as the plan and section drawing as quantitative representation. Both focused on the measured detail, which could be dimensions in the plan or section, and scientifically measured material and lighting in the rendering. Because of its precise and seductive appearance, rendering will always be functional and related to the capitalist culture of 
architecture, as it saw architecture as a commodity for selling purposes.

On the other hand, again, the discussion in the previous section can describe what is contained by images produced through the act of image-making. We can get an understanding of the statement, the intention, and the qualities of an unbuilt architectural space, even if it just an idea or statement of it. Furthermore, they are selective and could direct the attention of the observer to certain qualities of architecture.

Therefore, the act of image-making as a representation method and collage as a technique could be designated as qualitative representation; because it conveys beyond the tangible experience and qualities of an architectural artefact. The qualities contained are the reason why it has the ability to evoke its viewer to interpret more and to explore the hidden possibilities and potentials of an architectural space. Hence, perspectives produced through the act of image-making becomes the digital resurrection of the conventional architecture representation drawing.

\section{Acknowledgement}

The author would like to thank J, DEF, and his friends, who have been a great inspiration and encouragement for the author to take the enjoyment in finishing this paper in a pandemic and uncertainty situation. This paper is here in hope to be a part of the journey in thesis research for a master's degree and for those who interested, even people in general, to read.

\section{References}

Arkell, A. J. (2013) Drawing as Architecture: Representational Space Architecturally Transformed. Inquiry: The University of Arkansas Undergraduate Research Journal: Vol. 14, Article 4.

Bafna, S. (2008) How architectural drawings work - and what that implies for the role of representation in architecture. The Journal of Architecture, 13:5, 535-564.

Belting, H. (2005) Image, Medium, Body: A New Approach to Iconology. The University of Chicago Press Journal Vol. 31, No. 2, 302-319.

Bernath, D. (2007) The Intrusive Rendering: Dictation of Stereotypes and the Extra-Ordinary. Taiwan in Comparative Perspective, Vol. 1, 37-69.

Drozd, C., Meunier, V., Simonnot, N., and Hégron, G. (2009) What tools and modes of representation to reflect an architectural atmosphere? Conference on architectural visualisation - $9^{\text {th }}$ international conference, Faculty of Architecture, Brandenburg University of Technology.

Faruque, O. (1984) Graphic Communication As a Design Tool. New York: Van Nostrand Reinhold.

Forseth, K. (1991) Rendering the Visual Field - Illusion Becomes Reality. New York: Van Nostrand Reinhold.

Horn, K. J. (2017) Possibilities in Post-Digital Architecture. Thesis from the Architecture Program, University of Nebraska-Lincoln, 181.

Idi, D. B., and Khaidzir, K. A. M. (2010) Visualization in Design Process. Eenginering, Sciences \& Humanities (IGCESH), Malaysia.

Iskandar, I. (2005) Pergeseran Visualisasi Desain dari Manual ke Digital. Tesis Fakultas Seni Rupa dan Desain, Institut Teknologi Bandung, Bandung.
Kondor, Z. (2011) Representations and Cognitive Evolution. Towards an Anthropology of Pictorial Representation. Themenheft Homo Pictor und animal symbolicum, 48-71.

Krier, L. (2009) Drawing for architecture. Cambridge, MA: MIT Press.

Kurniati, F. (2015) Representasi Sebagai Bentuk Komunikasi dalam Arsitektur. Prosiding Temu Ilmiah IPLBI, 187-190.

Luce, K.M. (2009) Revolutions in Parallel: The Rise and Fall of Drawing in Architectural Design. University of Michigan.

Rahayu, G. A. F. dan Paramita A. (2015) Ambiguitas Pada Penerjemahan Kolase Sebagai Konsep dalam Perancangan Arsitektur. Department of Architecture, Universitas Indonesia.

Ratinam, M. (2012) Toward a Post-Digital Practice of Architectural Representation. Thesis from School of Architecture and Design, RMIT University, Melbourne.

Riahi, P. (2017) Expanding the boundaries of architectural representation. The Journal of Architecture, 22:5, 815-824.

Shields, J. A. E (2014) Collage and Architecture. New York: Routledge.

Suarez, L. (2015) Towards Experential Representation in Architecture. Journal of Architecture and Urbanism: Vol. 40(1), 47-58.

Tirta, A. A. (2009) Optimizing The Use of Computer in Architectural Presentation. Tesis Program Studi Magister Arsitektur, Universitas Katolik Parahyangan, Bandung. 